\title{
Low-expression of microRNA-203a in mid-gestational human fetal keratinocytes contributes to cutaneous scarless wound healing by targeting Tenascin-C
}

\author{
Meng Chen ${ }^{\mathrm{a}}$, Feng Zhao ${ }^{\mathrm{a}}$, Zhe Wang ${ }^{\mathrm{b}}$, Hongxin Lang ${ }^{\mathrm{a}}$, Kun Zhang ${ }^{\mathrm{a}}$, Tao Zhang ${ }^{\mathrm{a}}$, Rui Wang ${ }^{\mathrm{a}}$, \\ Ping Shi ${ }^{\mathrm{c}}$, Xining Pang ${ }^{\mathrm{a}, \mathrm{c}, *}$ \\ a Department of Stem Cells and Regenerative Medicine, Key Laboratory of Cell Biology, Ministry of Public \\ Health and Key Laboratory of Medical Cell Biology, Ministry of Education, China Medical University, \\ Shenyang, Liaoning, China \\ b Department of Pathology, Shengjing Hospital of China Medical University, Shenyang, Liaoning, China \\ c Shenyang Amnion Bioengineering Technology R \& D Center, Shenyang, Liaoning, China
}

*Corresponding author, e-mail: pangxining@126.com

Received 10 Jul 2019

Accepted 22 Feb 2020

\begin{abstract}
This study aimed to detect the roles of miRNA-203a in fetal cutaneous regeneration. The levels of miR203a in eight mid-gestational and ten late-gestational fetal epidermis tissues were detected by real-time qRT-PCR. The expressions of $\alpha$-SMA, TGF- $\beta 2$ and TGF- $\beta 3$ were detected by real-time qRT-PCR and Western blot. Moreover, the proliferation and migration abilities of keratinocytes (KCs) and fibroblasts (FBs) were examined by using MTS and transwell cell migration assays. Bioinformatics analyses, luciferase reporter and ELISA assays were used to examine the potential target genes. The results showed that miR-203a was dramatically up-regulated in late-gestational fetal epidermis tissues. MiR-203a inhibited the migration and proliferation abilities of KCs and FBs. Tenascin-C (TNC) was a direct target of miR-203a and miR-203a regulated TGF- $\beta$ isoforms and $\alpha$-SMA. It was concluded that the low-expression of miRNA-203a promoted KCs and FBs migration by targeting TNC and regulating TGF- $\beta$ isoforms and $\alpha$-SMA.
\end{abstract}

KEYWORDS: miR-203a, fetal skin, wound healing, fibrosis, TNC

\section{INTRODUCTION}

Chronic (hard-to-heal) wounds, are always worldwide problems from which patients suffer painfully [1]. Under normal condition, a highly orchestrated multi-step process triggered by injury leads to wound healing $[2,3]$. In the case of chronic wounds, this process is disrupted, leading to a prolonged inflammatory response, excessive scar formation and stalled healing $[4,5]$. Therefore, an appropriate therapeutic strategy for chronic wounds includes accelerating wound healing, inhibiting inflammation and preventing the hypertrophic scars formation.

Skin regeneration is the ideal way to repair tissues. At early- to mid-gestation, wounds of human fetal skin heal regeneratively (rapid healing with the absence of inflammation and scarring). However, a transition occurs after approximately the 28th week of gestation. After late-gestation, skin changes the manner of repair from regeneration to fibrosis [6-8]. Epidermal keratinocytes (KCs) play important roles during the transition. Fetal KCs of mid-gestation exhibit higher proliferation ability compared with KCs of late-gestational fetal skin. It has also been demonstrated that fetal KCs, especially fetal KCs of mid-gestation, significantly enhance abilities of fibroblast (FB) migration and FB proliferation and contribute to the extracellular matrix (ECM) which is related to skin regeneration $[9,10]$.

MicroRNAs (MiRNAs) are a large family of highly conserved small non-coding RNAs. They play important regulatory roles by regulating a vast number of protein-coding genes [11]. However, there is not much research which studies the role of miRNAs in fetal skin wound healing related to KCs. In this study, we investigated the differential expression of miRNAs between mid- and late-gestational fetal KCs and epidermis tissues and found miR-203a is dramatically up-regulated in late-gestational fetal KCs. Moreover, miR-203a had the effect of suppressing KC cell proliferation and migration. Overexpression of miR-203a in KCs significantly inhib- 
ited FB migration and up-regulated the expression of $\alpha$-SMA in FBs. The expressions of TGF- $\beta$ isoforms were also regulated by miR-203a. Furthermore, luciferase reporter assay and ELISA assay demonstrated that early in gestation, low-expression of miR-203a may promote scarless wound healing by targeting Tenascin-C (TNC).

\section{MATERIALS AND METHODS}

\section{Skin samples and cell culture}

Epidermis tissues were obtained from fetal skin samples. FBs were obtained from adult skin samples. The immortalized human keratinocyte cell line $\mathrm{HaCaT}$ was kindly provided by Chundi $\mathrm{He}$ and propagated in Dulbecco's Modified Eagle's medium (DMEM high glucose; Invitrogen Life Technologies, Carlsbad, CA, USA). This study was approved by the Ethics Committee of Shengjing Hospital affiliated with China Medical University. Written informed consent was obtained from all patients before participating.

Epidermis tissues and FBs were obtained as previously described $[11,12]$. Full-thickness skin specimens from the lower legs of miscarried fetuses were divided into two groups: the mid-gestation group (8 samples, gestational age 18-24 weeks) and the late-gestation group (10 samples, gestational age 29-36 weeks). Briefly, full-thickness skin samples were incubated at $4{ }^{\circ} \mathrm{C}$ overnight in Dispase II (Roche Applied Science, Indianapolis, IN). Epidermis was obtained after dermal components were removed by collagenase digestion. After mincing the dermal components, the isolated FBs were cultured in DMEM supplemented with $10 \%$ fetal bovine serum (Invitrogen Life Technologies) at $37^{\circ} \mathrm{C}$ in a humidified incubator of $5 \% \mathrm{CO}_{2}$.

\section{RNA isolation and real-time qRT-PCR}

Total RNA was extracted using the mir-Vana miRNA Isolation Kit (Ambion, Austin, TX, USA) according to the manufacturer's instructions. The concentration and purity of RNA were controlled by ultraviolet spectrophotometry (A260/A280>1.9) using a Nano-Photometer Ultraviolet/Vis spectrophotometer (Implen, Schatzbogen, Germany). The 3'termini of the RNA were polyadenylated using the Poly(A) Tailing Kit (ThermoFisher, USA). After that, RNA was extracted with phenol-chloroform and precipitated with ethanol.

Real-time qRT-PCR was used to confirm the expression levels of miR-203a, $\alpha$-SMA, TGF- $\beta 2$, TGF- $\beta 3$ and TNC. Reverse transcription was per-
Table 1 Real-time qRT-PCR primers.

\begin{tabular}{|c|c|}
\hline & Primer sequence $\left(5^{\prime}-3^{\prime}\right)$ \\
\hline RT-primer-1 & GCTGTCAACGATACGCTACGTAACGGCATGA \\
\hline & CAGTGTTTTTTTTTTTTTTTTTTTTTTTTA \\
\hline RT-primer-2 & GCTGTCAACGATACGCTACGTAACGGCATGA \\
\hline & CAGTGTTTTTTTTTTTTTTTTTTTTTTTTG \\
\hline RT-primer-3 & $\begin{array}{l}\text { GCTGTCAACGATACGCTACGTAACGGCATGA } \\
\text { CAGTGTTTTTTTTTTTTTTTTTTTTTTTTC }\end{array}$ \\
\hline hsa-miR-203a-Fa & GTGAAATGTTTAGGACCACTAG \\
\hline hsa-miR-203a-R ${ }^{b}$ & GCTGTCAACGATACGCTACGT \\
\hline U6 RNA-Fa & CGCTTCGGCAGCACATATAC \\
\hline U6 RNA-R ${ }^{b}$ & TTCACGAATTTGCGTGTCAT \\
\hline TGF- $\beta 2-F^{\mathrm{a}}$ & CAGCACACTCGATATGGACCA \\
\hline TGF- $\beta 2-R^{b}$ & CCTCGGGCTCAGGATAGTCT \\
\hline TGF- $\beta 3-F^{\mathrm{a}}$ & TGGCTGTTGAGAAGAGAGTCC \\
\hline TGF- $\beta 3-R^{b}$ & TGTCCACGCCTTTGAATTTG \\
\hline$\alpha-S M A-F^{a}$ & ACTCTTTCTACAATGAGCTTCGTG \\
\hline$\alpha-S M A-R^{b}$ & CATCTCCAGAGTCCAGCACG \\
\hline GAPDH-F & AGAAGGCTGGGGCTCATTTG \\
\hline GAPDH-R ${ }^{b}$ & AGGGGCCATCCACAGTCTTC \\
\hline TNC-Fa & CAAGTTCACAACAGACCTCG \\
\hline TNC-R $\mathrm{R}^{\mathrm{b}}$ & ACAATGACTTCCTTGACTGTG \\
\hline
\end{tabular}

${ }^{a}$ forward primer, ${ }^{b}$ reverse primer.

formed using a PrimeScript RT reagent Kit with gDNA Eraser (Takara Bio Inc., Shiga, Japan). Realtime qRT-PCR was performed on a 7500 Real-Time PCR system (Applied Biosystems, Foster City, CA, USA), using a SYBR Premix Ex Taq II Kit (Takara Bio Inc.). The PCR reactions used for the amplification of miRNAs were conducted at $95^{\circ} \mathrm{C}$ for $30 \mathrm{~s}$, followed by 45 cycles of $95^{\circ} \mathrm{C}$ for $5 \mathrm{~s}$ and $60^{\circ} \mathrm{C}$ for $34 \mathrm{~s}$. The U6 or GAPDH mRNA level, as an endogenous reference, was used for normalization. After the final cycle, a melting curve analysis was conducted within the range of 55$95^{\circ} \mathrm{C}$. The expression levels of miR-203a in lategestational fetal KCs relative to mid-gestational fetal KCs were calculated using the equation $2^{-\Delta \Delta C T}$ in which $\Delta \mathrm{C}_{\mathrm{T}}=\mathrm{C}_{\mathrm{T}}$ miRNA $-\mathrm{C}_{\mathrm{T}} \mathrm{U} 6$ [13]. The primers used for RT-PCR are given in Table 1 .

\section{Transfection}

HaCaT cells were plated 1 day before transfection. 2'-O-methyl oligonucleotides including miR-203a mimics and a stable negative control (NC) (GenePharma, China) were transfected using Lipofetamine 2000 (Invitrogen Life Technologies) according to the manufacturer's instructions. After $48 \mathrm{~h}$, the cells were harvested for further experiments.

\section{Co-culture of FBs with HaCaT}

The FBs were co-cultured with parental and transfected HaCaT cells in Transwell chambers $(3 \mu \mathrm{m}$ pore size; Corning Costar, USA) for $96 \mathrm{~h}$. FBs were plated in 24-well tissue culture plates. The parental $\mathrm{HaCaT}$ cells or NC/miR-203a-transfected HaCaT 
cells were placed in the upper chamber. After that, the FBs were harvested for further experiments.

\section{Cell migration assay}

We used the transwell cell migration assay to estimate the migration ability of HaCaT cell line in vitro. The bottom of the culture inserts ( $8 \mu \mathrm{m}$ pores) in 24-well tissue culture plates (Transwell, Corning, USA) was coated with serum-free medium at $37^{\circ} \mathrm{C}$ for 1 h. $5 \times 10^{4}$ cells treated with mitomycin-C to inhibit cell proliferation were harvested by trypsinization, washed with serum-free RPMI-medium to $5 \times 10^{5} / \mathrm{ml}$ and placed in the upper chamber. The lower chamber contained 10\% FBS for use as a chemoattractant. After $48 \mathrm{~h}$ of incubation at $37^{\circ} \mathrm{C}$ with $5 \% \mathrm{CO}_{2}$, the number of cells that had migrated to the basal side of the membrane was quantified by counting 16 independent symmetrical visual fields under the microscope. The cell morphology was observed by staining with H\&E (Hematoxylin and Eosin).

\section{Quantification of cell proliferation}

The MTS (CellTiter 96 Aqueous, WI, USA) assay was used to estimate the proliferation ability of FBs and $\mathrm{HaCaT}$ cells. In all, $5 \times 10^{3}$ cells were seeded into 96-well culture plates for 24, 48, 72 and $96 \mathrm{~h}$, separately. After incubating with $20 \mu \mathrm{l}$ of MTS for $2 \mathrm{~h}$ at $37^{\circ} \mathrm{C}$, absorbance was measured at a wavelength of $495 \mathrm{~nm}$ using an iMARK microplate reader (Bio-Rad, CA, USA).

\section{In vitro scratch wound healing assay}

In vitro scratch wound closure assay was performed as described previously [9]. Briefly, FBs were cultured in 6-well microplates under normal culture conditions and allowed to reach maximum confluence. A wound line was created by scratching the plates with a $10 \mu \mathrm{l}$ micropipette tip. Microplates were then washed with DMEM three times for 10 min each wash followed by culture in starving medium (DMEM containing 1\% FBS). The status of the scratch wounds was monitored using an inverted microscope at $0,6,12$ and $24 \mathrm{~h}$, then representative images were collected. The results are presented as the percentage of wound healing, calculated as follows:

$$
\frac{\text { wound area (initial) }- \text { wound area (final) }}{\text { wound area (initial) }} \times 100
$$

\section{Western blot analysis}

Total protein was extracted using a Total Protein Extraction Kit (KeyGen, Nanjing, China). Each
Table 2 PCR primers for amplification of TNC $3^{\prime} \mathrm{UTR}$ segment.

\begin{tabular}{ll}
\hline & Primer sequence $\left(5^{\prime}-3^{\prime}\right)$ \\
\hline TNC-F $^{\mathrm{a}}$ & GCTCTAGATGACAACATCGCAATAG \\
TNC- $^{\mathrm{b}}$ & GCTCTAGATGGAAAGAAAGATCTCTTG \\
\hline
\end{tabular}

${ }^{\mathrm{a}}$ forward primer, ${ }^{\mathrm{b}}$ reverse primer.

sample was separated by $12 \%$ SDS polyacrylamide gels and transferred to polyvinylidene difluoride membranes (Millipore, Billerica, MA, USA). After incubation with $5 \%$ bovine serum albumin, the membranes were incubated at $4{ }^{\circ} \mathrm{C}$ overnight with primary antibodies against TGF- $\beta 2$ (ImmunoWay, USA), TGF- $\beta 3$ and Gapdh (Santa Cruz Biotechnology, CA, USA) and then incubated with horseradish peroxidase-conjugated secondary antibody. The antigen-antibody complexes were visualized using an ECL Kit (Pierce, Rockford, IL, USA). Quantification of protein was carried out using FluorChem 2.01 (Alpha Innotech, San Leandro, CA, USA).

\section{Bioinformatics analyses and luciferase reporter assays}

We used microrna.org online software (www. microrna.org/microrna/getMirnaForm.do) to predict miRNA target genes. Among all the genes that were compiled, we determined TNC which may contribute to scarless wound healing.

The $3^{\prime}$ UTR segment of TNC containing the miR203a binding sites was amplified by PCR, primers were shown in Table 2. After that, the $3^{\prime}$ UTR segment was inserted into the pGL3-control vector (Promega, USA) using the XbaI site. HaCaT cells were transfected in a 24-well plate using Lipofetamine 2000 with $0.8 \mu \mathrm{g}$ of the firefly luciferase report vector and $0.08 \mu \mathrm{g}$ of the control vector containing Renilla luciferase, pRL-TK (Promega). For each well, 20 pM hsa-miR-203a or NC was used for transfection. After 24 h, Firefly and Renilla luciferase activities were measured consecutively on a Centro LB 960 (Berthold, USA) using a dualluciferase reporter assay (Promega).

\section{Detection of TNC expression}

For TNC detection, $5 \times 10^{5} \mathrm{HaCaT}$ cells were seeded in the 6-well microplates under normal culture conditions for $72 \mathrm{~h}$, then the culture medium was collected. After centrifugation, concentrations of TNC in the cell culture medium were evaluated by ELISA Kits (R\&D, Minneapolis, USA) according to the manufacturer's instructions. 


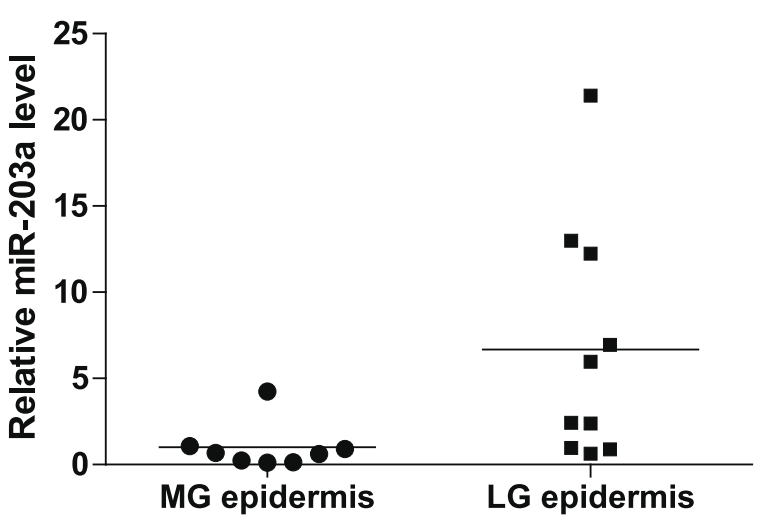

Fig. 1 The expression of miR-203a in fetal epidermis tissues detected by real-time qRT-PCR. MG = Mid-gestation, $\mathrm{LG}=$ Late-gestation.

\section{Statistical analysis}

The relative expressions of miRNAs and TNC detected by qRT-PCR were analyzed by the nonparametric $t$-test: Mann-Whitney $U$ test. The other results were presented as the mean \pm SD from at least three separate experiments and analyzed using the Student's $t$-test and $p$ values less than 0.05 were considered statistically significant. All the statistical analyses were performed using SPSS 16.0 software.

\section{RESULTS}

MiR-203a is up-regulated in the late-gestational fetal epidermis tissues

To determine whether miR-203a expression in epidermis tissues is associated with different gestational ages, we examined miR-203a expression in a cohort consisting of 8 mid-gestational and 10 late-gestational fetal epidermis tissues using realtime qRT-PCR. A significant difference of miR203a expression was found between mid- and lategestational fetal epidermis tissues (Fig. 1). The nonparametric test revealed that the expression of miR-203a was significantly higher in the lategestational fetal epidermis group compared with the mid-gestational fetal epidermis group (6.68 \pm 6.89 folds, $p<0.05$ ).

\section{MiR-203a suppresses KC proliferation and migration in vitro}

The significant reduction of miR-203a expression in mid-gestational fetal epidermis samples prompted us to explore the possible biological significance of miR-203 in KCs. As an initial step, HaCaT cell line was transfected with hsa-miR-203a mimics or NC. Transfection efficiency was perfect (Fig. 2a).
MTS assay was used to estimate the effect of miR-203a on the proliferation of HaCaT cells. According to the results, we found the HaCaT cells, which were transiently transfected with miR-203a mimics, had a significant growth inhibition at different degrees (Fig. 2b).

We estimated the effect of miR-203a on the migration of the HaCaT cells using transwell cell migration assay. The numbers of miR-203atransfected HaCaT cells $(14.563 \pm 4.966, p<0.01)$ passing through the membrane were significantly lower than the numbers of NC-transfected HaCaT cells $(30.438 \pm 11.599)$ or parental HaCaT $(34.563 \pm 13.327)$ cells. There was no significant difference between NC-transfected HaCaT cells and parental HaCaT cells ( $p=0.28$, Fig. $2 c d$ ). The data demonstrated that over-expression of miR-203a obviously inhibited the migration of $\mathrm{HaCaT}$ cell line.

\section{Effect of miR-203a in KCs on FB proliferation and migration in co-culture}

To identify the role of miR-203a in KCs on the proliferation and migration of FBs in wound healing, images of wounds on FB monolayer were captured at $0,6,12,24 \mathrm{~h}$ after scratching. The results showed that FB migration into the wound area was decelerated in co-culture with KCs of which miR203a was over-expressed (Fig. 3ab).

Moreover, we compared the differences in the stimulatory effect of HaCaT on FBs by transwell coculture with parental and transfected HaCaT cells. After $96 \mathrm{~h}$, the proliferation of FBs was estimated using MTS assay. The result showed that overexpression of miR-203a in HaCaT cells has no significant effects on FB proliferation (Fig. 3c).

\section{MiR-203a regulates TGF- $\beta$ isoforms and $\alpha$-SMA}

We detected the mRNA and protein levels of the TGF- $\beta$ isoforms in HaCaT cells by real-time qRTPCR and Western blot. After being transfected with miR-203a mimics, HaCaT cells expressed more TGF$\beta 2$ and less TGF- $\beta 3$ compared with cells transfected with NC (Fig. 4ab).

Moreover, we detected the expression of $\alpha$ SMA in FBs which had been co-cultured with parental and transfected HaCaT cells for $96 \mathrm{~h}$. The mRNA level of $\alpha$-SMA in FBs co-cultured with miR-203a-transfected HaCaT cells was significantly higher than FBs co-cultured with NC-transfected or parental HaCaT cells (Fig. 5). 

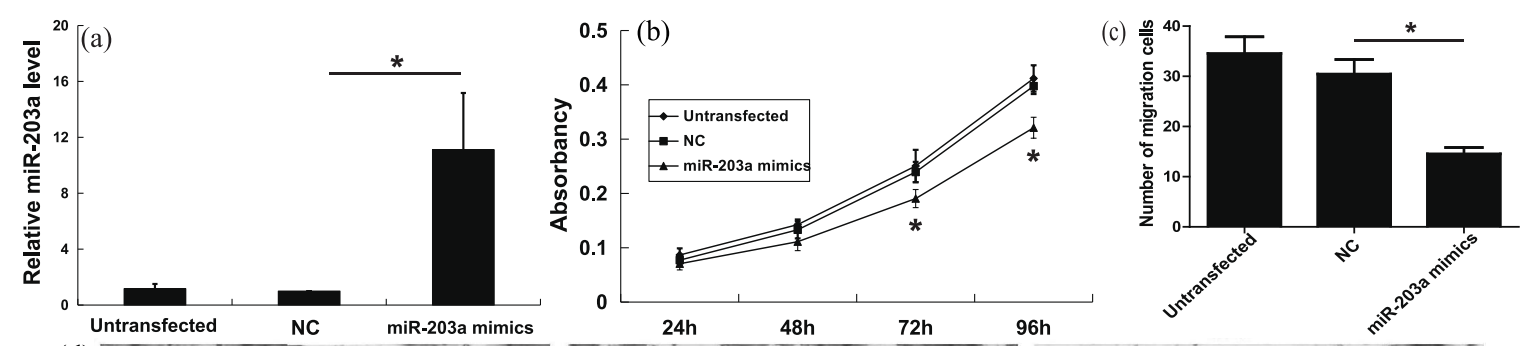

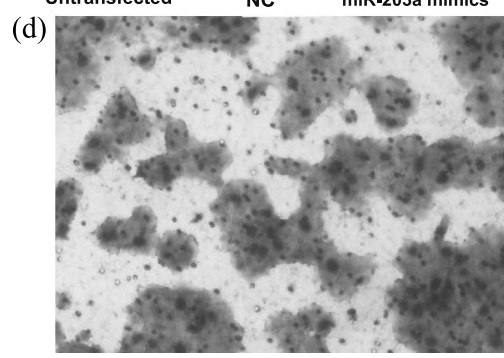

Untransfected

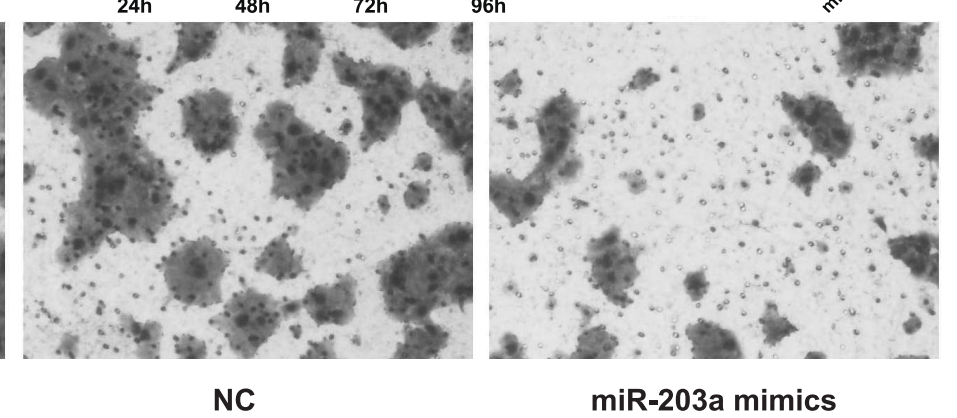

Fig. 2 Over-expression of miR-203a suppresses KC migration and proliferation. (a) Transfection efficiency of hsamiR-203a mimics in HaCaT cells measured by qRT-PCR. (b) MTS proliferation assay in parental and transfected HaCaT cells. (c) The numbers of HaCaT cells under different treatments. The number of cells was counted in 16 independent symmetrical visual fields under the microscope $(200 \times$ original magnification) from 3 independent experiments. (d) Representative photomicrographs of transwell results for HaCaT cells taken under $100 \times$ original magnification. $\mathrm{NC}=$ Negative control, * refers to statistical significance between groups $(p<0.05)$.

\section{Target binding to miR-203a suppresses the expression of TNC in KCs}

For miRNA target gene prediction, we used miRanda online software. Among all the genes that were potentially targeted by miR-203a, we found TNC may contribute to the abilities of cell migration and proliferation (Fig. 6a). For further evidence, we constructed luciferase reporter pGL3-TNC-3'UTR. The reporter was transfected in HaCaT cells. Luciferase activity of the reporter was significantly suppressed in miR-203a-transfected HaCaT cells compared with NC-transfected $\mathrm{HaCaT}$ cells normalized to a control vector containing Renilla luciferase, pRL-TK (Fig. 6b). These results showed that TNC are negatively regulated by miR-203a in KCs.

The level of the TNC in ECM plays an important role in scarless wound healing. Thus, we examined the TNC protein levels in the culture medium of parental and transfected HaCaT cells by ELISA, and observed a clear reduction in the expression of the TNC in miR-203a-transfected HaCaT cells $(18.47 \pm 6.24 \%, p<0.05)$ compared with NC-transfected HaCaT cells (Fig. 6c). These results demonstrated that miR-203a may suppress the expression of TNC in KCs.

\section{Tenascin- $C$ is down-regulated in the late-gestational fetal epidermis tissues}

To determine whether the expression of TNC in epidermis tissues is different in mid- and lategestational ages, we detected TNC expression in six mid-gestational and eight late-gestational fetal epidermis tissues using real-time qRT-PCR. A significant difference of TNC expression was found between mid- and late-gestational fetal epidermis tissues (Fig. 7). The nonparametric test revealed that the level of TNC was significantly downregulated in the late-gestational fetal epidermis group compared with the mid-gestational fetal epidermis group $(0.16 \pm 0.13$-fold, $p<0.05)$.

\section{DISCUSSION}

Recently, the role of miRNAs in scarless wound healing was uncovered. Previously [14], we detected the expression of miRNAs in human fetal KCs of different gestational ages using next generation sequencing and found miR-203a is up-regulated in the late-gestational fetal KCs. The expression of miR-203a is more than 100-fold higher in skin compared to other organs. Since miR-203a is a skin-specific expressed miRNA [15], its dynamic expression attracted our attention greatly. To further demonstrate whether the expression of miR-203a 

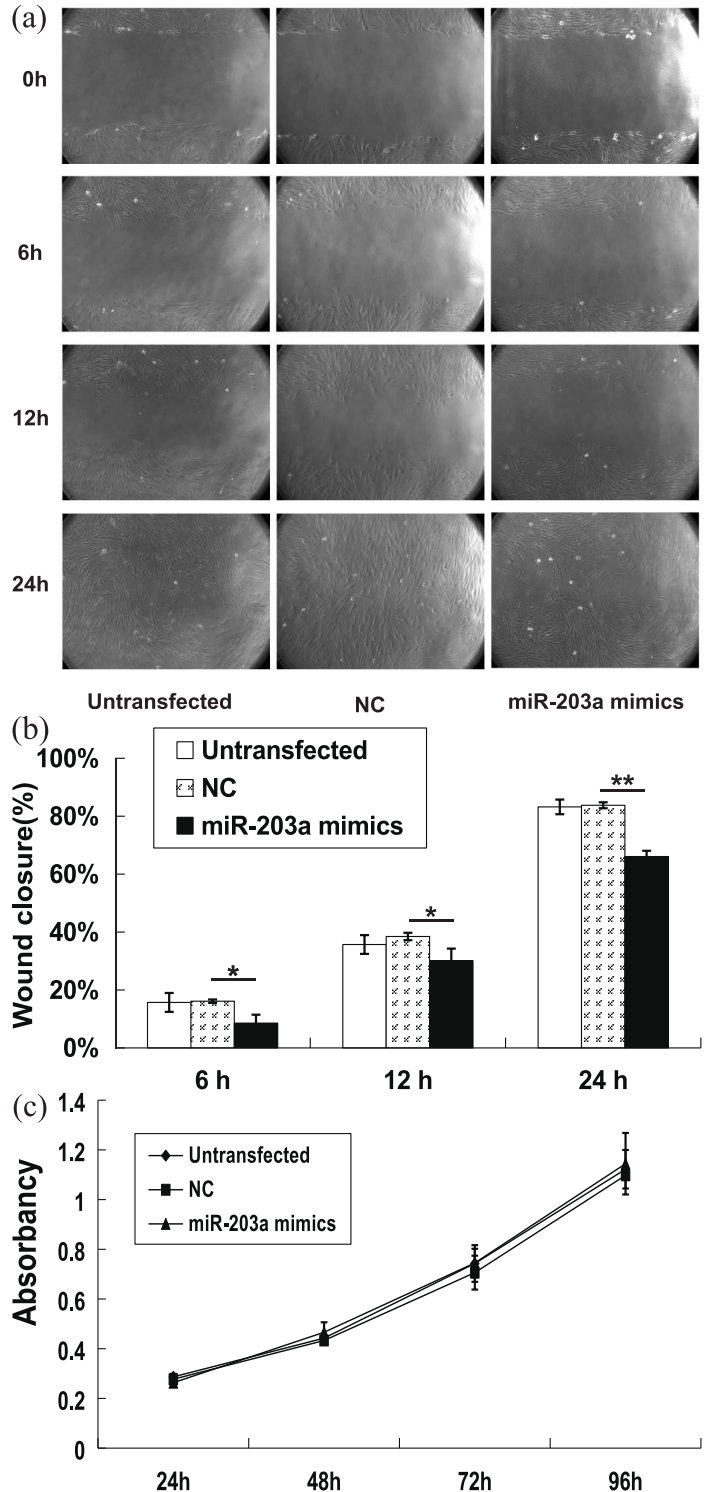

Fig. 3 Effects of miR-203a-transfected KCs on the migration and proliferation of FBs. (a) Representative photomicrographs of FB migration into the scratch wound after 0 , 6, 12, and 24 h. (b) Rate of movement after 6, 12 and $24 \mathrm{~h} ; *$ and ** refer to statistical significance between groups ( $p<0.05$ and $p<0.01$, respectively). (c) MTS proliferation assay in $\mathrm{FBs}$ after co-culture with parental or transfected HaCaT cells for $96 \mathrm{~h}$. NC $=$ Negative control.

in fetal KCs is associated with different gestational ages, we examined miR-203a expression in eight mid-gestational and ten late-gestational fetal epidermis tissues by qRT-PCR and found a significant over-expression of miR-203a (more than 6-folds) in late-gestational fetal epidermis tissues. MiR-203a
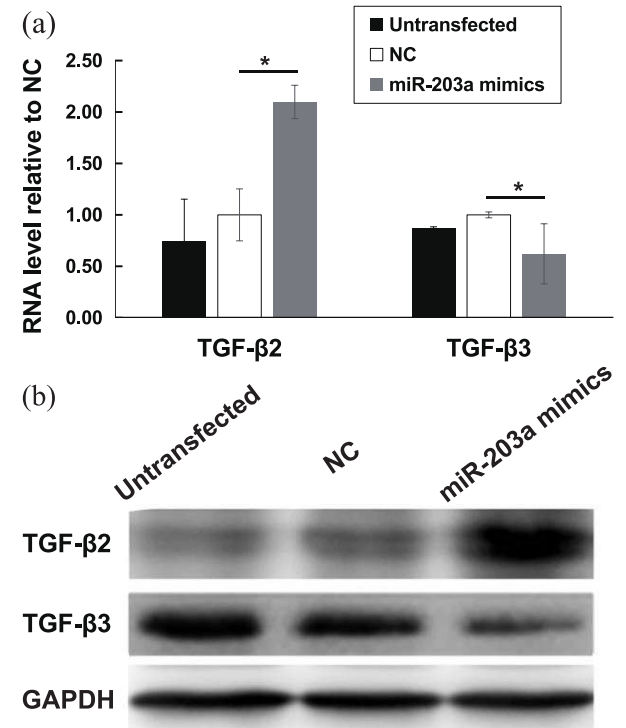

Fig. 4 miR-203a regulates TGF- $\beta$ isoforms. (a) Realtime qRT-PCR showing levels of RNA for TGF- $\beta 2$ and TGF$\beta 3$ proteins in HaCaT cells under different treatments; * refers to statistical significance between groups $(p<$ 0.05). (b) Western blotting showing levels of TGF- $\beta 2$ and TGF- $\beta 3$ proteins in HaCaT cells under different treatments. $\mathrm{NC}=$ Negative control.

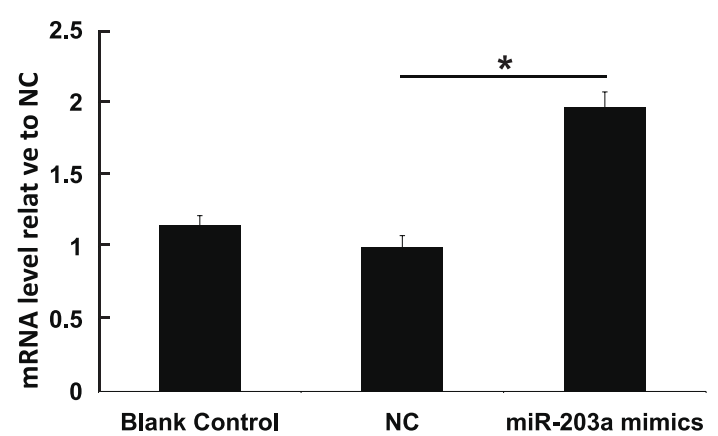

Fig. 5 miR-203a up-regulates $\alpha$-SMA in FBs. Real-time qRT-PCR showing the mRNA level of $\alpha$-SMA in FBs. Blank control refers to the group that FBs co-cultured with parental HaCaT cells; NC = Negative control; * refers to statistical significance between groups $(p<0.05)$.

showing dynamic expression at different gestational ages in fetal KCs suggests that miR-203a may play a role in fetal cutaneous repair.

MiR-203a is a significant tumor suppressor in various cancers including nasopharyngeal carcinoma, prostate cancer, gastric cancer, and lung cancer [16-19]. It inhibits proliferation, invasion and migration abilities of many kinds of cells [20, 
(a)

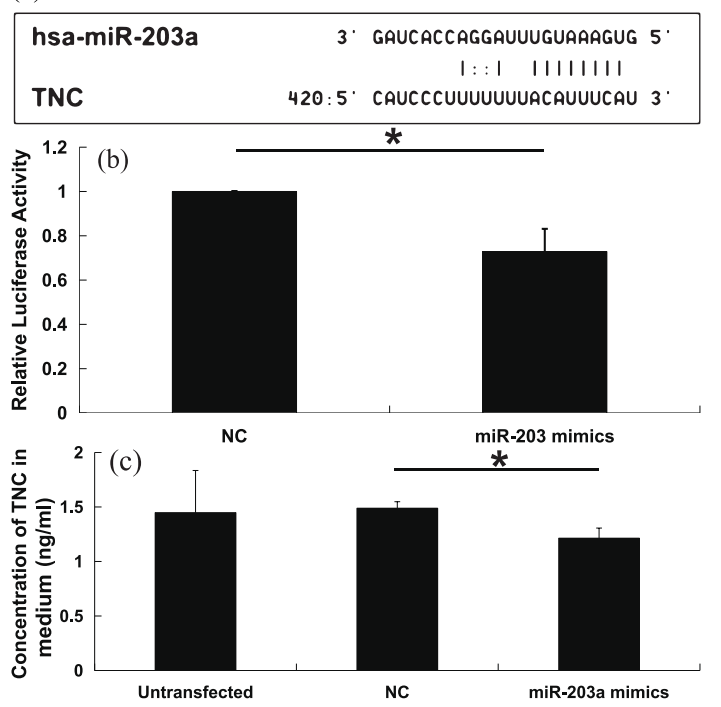

Fig. 6 TNC was negatively regulated by miR-203a in HaCaT cells. (a) TNC was a potential target of miR203a predicted by microrna.org online software. (b) Data present the relative repression of firefly luciferase expression standardized to a transfection control, renilla luciferase. Relative luciferase activity of luciferase reporter pGL3-TNC-3'UTR $(72.88 \pm 10.26 \%, p<0.05)$ was measured in miR-203a-transfected HaCaT cells compared with NC-transfected HaCaT cells. (c) The results of ELISA analysis on the expression of TNC in HaCaT cells after miR-203a and NC transfection. NC = Negative control, * refers to statistical significance between groups $(p<$ 0.05).

21]. During mammalian cutaneous wound healing, re-epithelization by KC proliferation and migration is a crucial step for covering the denuded dermal surface [22]. Failure of re-epithelization to maintain the barrier may slow down the process of wound healing. Is the low-expression of miR-203a associated with KC cell activities? We estimated the effect of miR-203a on the proliferation and migration of the epithelial cell line HaCaT derived from adult human skin, and found miR-203a significantly suppresses KC proliferation and migration. These suggest that miR-203a may suppress proliferation and migration of KCs and be involved in cutaneous wound healing.

FBs play key roles in fetal cutaneous scarless wound healing [23]. Many studies have demonstrated that altering the phenotype of FBs has the effect of controlling scar formation [24]. KCs exert a positive effect on proliferation and migration in FBs [25]. In order to see if the dynamic expression

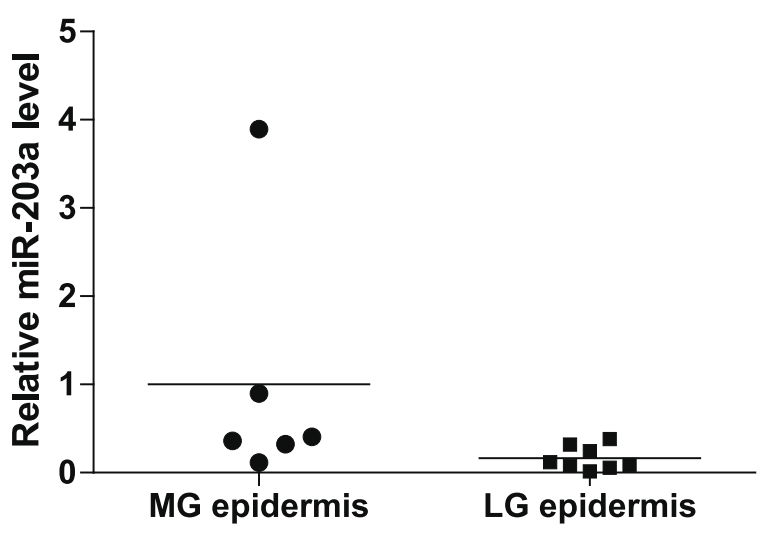

Fig. 7 The expression of TNC in fetal epidermis tissues detected by real-time qRT-PCR. Relative expression of TNC in 14 fetal epidermis samples. Quantification of mRNA was measured by qRT-PCR. The nonparametric test revealed that the expression of TNC in fetal epidermis tissues was associated with gestational ages $(p<0.05)$. MG = Mid-gestation, $\mathrm{LG}=$ Late-gestation .

of miR-203a in KCs have effects on FBs, we studied the FB proliferation and migration abilities after coculture with parental and transfected $\mathrm{HaCaT}$ cells and found that over-expression of miR-203a in HaCaT cells has no significant effects on FB proliferation but significantly inhibits FBs migration. MiR203a inhibiting FBs migration may inhibit wound healing and lead to scar formation.

TGF- $\beta$ is a multifunctional growth factor family that plays pleiotropic effects on wound healing by regulating ECM production, cell proliferation, migration and inflammatory reaction $[26,27]$. TGF$\beta 1$ and TGF- $\beta 2$ are key factors which lead to scar formation. In keloid tissues, they are highly expressed [28]. During the fetal stage, skin expressing high levels of TGF- $\beta 3$ and low levels of TGF- $\beta 1$ and TGF- $\beta 2$ leads to scarless wound healing [29]. TGF$\beta 3$ restricts scarring, improves collagen organization and scarless wound healing in vivo while simultaneously enhancing keratinocyte migration [30]. In this study, using real-time qRT-PCR and Western blot, we found that miR-203a-transfected HaCaT cells expressed more TGF- $\beta 2$ and less TGF- $\beta 3$. It suggests that miR-203a have a significant influence on scarless wound healing, which is associated with TGF- $\beta$ isoforms. Excessive hyperplasia of myofibroblasts which express $\alpha$-SMA is another important factor in scar formation [31]. MiR-203a-transfected $\mathrm{HaCaT}$ cells up-regulating the expression of $\alpha$-SMA provide significant evidence of miR-203a inducing FBs differentiation to myofibroblasts. 
Further analyses were conducted to elucidate the molecular mechanism underlying miR-203a acting as a cell proliferation and migration suppressor. Using a miRanda online software, we predict TNC, an ECM glycoprotein, may be a target of miR-203a. TNC is associated with epithelial-mesenchymal interactions, wound healing and cell motility in the embryo [32,33]. Recent studies have shown that TNC is abundantly present in the dermal component during embryogenesis and wound healing [34]. It promotes proliferation and migration of various cells including KCs [35-37]. Besides, TNC promotes FBs migration and shows earlier deposition in fetal wounds, which contributes to the ability to re-epithelize rapidly with a reduced presence of inflammatory cells and fetal cutaneous scarless wound healing $[38,39]$.

In this study, we used luciferase reporter and ELISA assays to confirm that TNC is a target of miR-203a in KCs. The results demonstrated that miR-203a may act as a proliferation and migration suppressor by targeting TNC.

In conclusion, our study showed that miR203a is dramatically up-regulated in late-gestational fetal epidermis tissues. MiR-203a has the effect of suppressing KC cell proliferation and migration and targeting TGF- $\beta$ isoforms. Moreover, overexpression of miR-203a in KCs significantly inhibits FB migration and promotes FBs differentiation to myofibroblasts. Early in gestation, low-expression of miR-203a may promote scarless wound healing by regulating TGF- $\beta$ isoforms and targeting TNC.

Acknowledgements: This work was supported by the Program for National Basic Research Program of China (No. 2012CB518103), National Science Foundation of China (No. 81370883 and 81601692), National Key Research and Development Project (No. 2017TFC1103300), Shenyang Key Laboratory Project (No. F15-157-1-00), and New Teacher Foundation of China Medical University (No. XZR 20160023).

\section{REFERENCES}

1. Bayer A, Tohidnezhad M, Lammel J, Lippross S, Behrendt P, Klüter T, Pufe T, Jahr H, et al (2017) Platelet-released growth factors induce differentiation of primary keratinocytes. Mediators Inflamm 2017, ID 5671615.

2. Yang X, Wang J, Guo SL, Fan KJ, Li J, Wang YL, Teng Y, Yang X (2011) miR-21 pro-motes keratinocyte migration and re-epithelialization during wound healing. Int J Biol Sci 7, 685-690.

3. Jin Y, Tymen SD, Chen D, Fang ZJ, Zhao Y, Dragas D, Dai Y, Marucha PT, et al (2013) MicroRNA-99 fam- ily targets AKT/mTOR signaling pathway in dermal wound healing. PLoS One 8, ID e64434.

4. Kalan L, Zhou M, Labbie M, Willing B (2017) Measuring the microbiome of chronic wounds with use of a topical antimicrobial dressing - A feasibility study. PLoS One 12, ID e187728.

5. Hesketh M, Sahin KB, West ZE, Murray RZ (2017) Macrophage phenotypes regulate scar formation and chronic wound healing. Int J Mol Sci 18, ID E1545.

6. Chen W, Fu X, Ge S, Sun T, Zhou G, Jiang D, Sheng $\mathrm{Z}$ (2005) Ontogeny of expression of transforming growth factor-beta and its receptors and their possible relationship with scarless healing in human fetal skin. Wound Repair Regen 13, 68-75.

7. Samuels P, Tan AKW (1999) Fetal scarless wound healing. J Otolaryngol 28, 296-302.

8. Dang C, Ting K, Soo C, Longaker MT, Lorenz HP (2003) Fetal wound healing current perspectives. Clin Plast Surg 30, 13-23.

9. Wang Z, Liu X, Zhang D, Wang X, Zhao F, Zhang $\mathrm{T}$, Wang R, Lin $\mathrm{X}$, et al (2015) Phenotypic and functional modulation of 20-30 year old dermal fibroblasts by mid- and late-gestational keratinocytes in vitro. Burns 41, 1064-1075.

10. Wang Z, Liu X, Zhang D, Wang X, Zhao F, Shi P, Pang $X$ (2015) Co-culture with human fetal epidermal keratinocytes promotes proliferation and migration of human fetal and adult dermal fibroblasts. Mol Med Rep 11, 1105-1110.

11. Zhao F, Lang H, Wang Z, Zhang T, Zhang D, Wang $\mathrm{R}$, Lin X, Liu X, et al (2019) Human novel microRNA Seq-915_x4024 in keratinocytes contributes to skin regeneration by suppressing scar formation. Mol Ther Nucleic Acids 14, 410-423.

12. Liu X, Wang Z, Wang R, Zhao F, Shi P, Jiang Y, Pang $X$ (2013) Direct comparison of the potency of human mesenchymal stem cells derived from amnion tissue, bone marrow and adiposetissue at inducing dermal fibroblast responses to cutaneous wounds. Int $\mathrm{J} \mathrm{Mol}$ Med 31, 407-415.

13. Livak KJ, Schmittgen TD (2001) Analysis of relative gene expression data using real-time quantitative PCR and the 2(-Delta Delta C(T)) method. Methods 25, 402-408.

14. Zhao F, Wang Z, Lang H, Liu X, Zhang D, Wang X, Zhang T, Wang R, et al (2019) Dynamic expression of novel MiRNA candidates and MiRNA-34 family members in early- to mid-gestational fetal keratinocytes contributes to scarless wound healing by targeting the TGF- $\beta$ Pathway. PLoS One 10, ID e126087.

15. Bostjancic E, Glavac D (2008) Importance of microRNAs in skin morphogenesis and diseases. Acta Dermatovenerol Alp Pannonica Adriat 17, 95-102.

16. Jiang N, Jiang X, Chen Z, Song X, Wu L, Zong D, Song D, Yin L, et al (2017) MiR-203a-3p suppresses cell proliferation and metastasis through inhibiting 
LASP1 in nasopharyngeal carcinoma. $J$ Exp Clin Cancer Res 36, ID 138.

17. Tian X, Tao F, Zhang B, Dong JT, Zhang Z (2018) The miR-203/SNAI2 axis regulates prostate tumor growth, migration, angiogenesis and stemness potentially by modulating GSK-3 $\beta / \beta$-CATENIN signal pathway. IUBMB Life 70, 224-236.

18. Yang H, Wang L, Tang X, Bai W (2017) miR-203a suppresses cell proliferation by targeting E2F transcription factor 3 in human gastric cancer. Oncology Letters 14, 7687-7690.

19. Chi $Y$, Jin Q, Liu X, Xu L, He X, Shen Y, Zhou Q, Zhang J, et al (2017) miR-203 inhibits cell proliferation, invasion, and migration of non-small-cell lung cancer by downregulating RGS17. Cancer Sci 108, 2366-2372.

20. Zang J, Hui L, Yang N, Yang B, Jiang X (2018) Downregulation of miR-203a disinhibits Bmil and promotes growth and proliferation of keratinocytes in cholesteatoma. Int J Med Sci 15, 447-455.

21. Zheng XB, Chen XB, Xu LL, Zhang M, Feng L, Yi PS, Tang JW, Xu MQ (2017) miR-203 inhibits augmented proliferation and metastasis of hepatocellular carcinoma residual in the promoted regenerating liver. Cancer Sci 108, 338-346.

22. Pastar I, Stojadinovic O, Yin NC, Ramirez H, Nusbaum AG, Sawaya A, Patel SB, Khalid L, et al (2014) Epithelialization in wound healing: A comprehensive review. Adv Wound Care 3, 445-464.

23. Thulabandu V, Chen D, Atit RP (2018) Dermal fibroblast in cutaneous development and healing. WIREs Dev Biol 7, ID e307.

24. Parekh A, Hebda PA (2017) The contractile phenotype of dermal fetal fibroblasts in scarless wound healing. Curr Pathobiol Rep 5, 271-277.

25. Carr MJ, Li Y, Rezakhanlou AM, Ghahary A (2017) Keratinocyte-releasable factors stimulate the expression of granulocyte colony-stimulating factor in human dermal fibroblasts. J Cell Biochem 118, 308-317.

26. Morikawa M, Derynck R, Miyazono K (2016) TGF$\beta$ and the TGF- $\beta$ family: context-dependent roles in cell and tissue physiology. Cold Spring Harb Perspect Biol 8, ID a021873.

27. Koivisto L, Heino J, Häkkinen L, Larjava H (2014) Integrins in wound healing. Adv Wound Care 3, 762-783.

28. Lee TY, Chin GS, Kim WJ, Chau D, Gittes GK, Longaker MT (1999) Expression of transforming growth factor beta 1, 2, and 3 proteins in keloids. Ann Plast Surg 43, 179-184.
29. Hsu M, Peled ZM, Chin GS, Liu W, Longaker MT (2001) Ontogeny of expression of transforming growth factor- $\beta 1$ (TGF- $\beta 1$ ), TGF- $\beta 3$, and TGF- $\beta$ receptors I and II in fetal rat fibroblasts and skin. Plast Reconstr Surg 107, 1795-1796.

30. Lichtman MK, Otero-Vinas M, Falanga V (2016) Transforming growth factor beta (TGF- $\beta$ ) isoforms in wound healing and fibrosis. Wound Repair Regen 24, 215-222.

31. Zhou Z, Shu B, Xu Y, Liu J, Wang P, Chen L, Zhao J, Liu X, et al (2018) microRNA-203 modulates wound healing and scar formation via suppressing hes1 expression in epidermal stem cells. Cell Physiol Biochem 49, 2333-2347.

32. Chiquet-Ehrismann R, Orend G, Chiquet M, Tucker RP, Midwood KS (2014) Tenascins in stem cell niches. Matrix Biol 37, 112-123.

33. Nishio $T$, Kawaguchi $S$, Yamamoto $M$, Iseda $T$, Kawasaki T, Hase T (2005) Tenascin-C regulates proliferation and migration of cultured astrocytes in a scratch wound assay. Neuroscience 132, 87-102.

34. Tucker RP, Ferralli J, Schittny JC, Chiquet-Ehrismann $R$ (2013) Tenascin-C and tenascin-W in whisker follicle stem cell niches: possible roles in regulating stem cell proliferation and migration. J Cell Sci 126, 5111-5115.

35. Akbareian SE, Nagy N, Steiger CE, Mably JD, Miller SA, Hotta R, Molnar D, Goldstein AM (2013) Enteric neural crest-derived cells promote their migration by modifying their microenvironment through tenascinC production. Dev Biol 382, 446-456.

36. Nemoto M, Kizaki K, Yamamoto $Y$, Oonuma $T$, Hashizume K (2013) Tenascin-C expression in equine tendon-derived cells during proliferation and migration. J Equine Sci 24, 17-23.

37. Itoh S, Yamaoka N, Kamoshida G, Takii T, Tsuji T, Hayashi H, Onozaki K (2013) Staphylococcal superantigen-like protein 8 (SSL8) binds to tenascin $\mathrm{C}$ and inhibits tenascin C-fibronectin interaction and cell motility of keratinocytes. Biochem Biophys Res Commun 433, 127-132.

38. Harman RM, He MK, Zhang S, Vav De Walle GR (2018) Plasminogen activator inhibitor-1 and tenascin-C secreted by equine mesenchymal stromal cells stimulate dermal fibroblast migration in vitro and contribute to wound healing in vivo. Cytotherapy 20, 1061-1076.

39. Yates CC, Hebda P, Wells A (2012) Skin wound healing and scarring: fetal wounds and regenerative restitution. Birth Defects Res C Embryo Today 96, 325-333. 